$\begin{array}{ll}\text { Research Square } & \begin{array}{l}\text { Preprints are preliminary reports that have not undergone peer review. } \\ \text { They should not be considered conclusive, used to inform clinical practice, } \\ \text { or referenced by the media as validated information. }\end{array}\end{array}$

\title{
Microbiome analysis of bile samples in patients with choledocholithiasis and hepatobiliary disorders
}

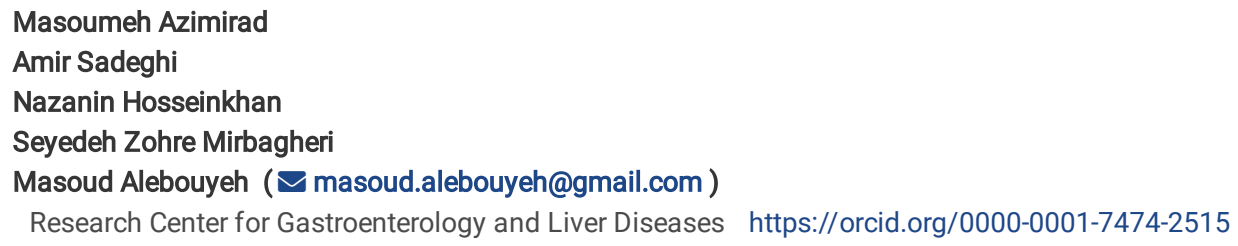

Masoumeh Azimirad

Amir Sadeghi

Nazanin Hosseinkhan

Seyedeh Zohre Mirbagheri

Masoud Alebouyeh ( $\nabla$ masoud.alebouyeh@gmail.com)

Research Center for Gastroenterology and Liver Diseases https://orcid.org/0000-0001-7474-2515

\section{Research Article}

Keywords: Microbiota, Bile, Infections, Choledocholithiasis, Pancreatic cancer, Sphincterotomy, Mothur, Kraken, 16S rDNA

Posted Date: March 2nd, 2022

DOI: https://doi.org/10.21203/rs.3.rs-1398466/v1

License: (9) (7) This work is licensed under a Creative Commons Attribution 4.0 International License. Read Full License 


\section{Abstract}

Involvement of bacteria in the pathogenesis of biliary tract is largely unknown. In this study, we investigated differences in the microbiota of biliary tissue among adult patients with choledocholithiasis during endoscopic retrograde cholangiography (ERCP). 16S rDNA sequencing of bile samples, culture, and data of the medication history, underlying diseases and liver function tests were used for interpretation of differences observed in composition of bacterial taxa detected in the patients. The most common phyla found among the bile samples were included Proteobacteria, Firmicutes, Actinobacteria, Bacteroidetes, Cyanobacteria/Chloroplast, Fusobacteria, Acidobacteria, and Deinococcus-Thermus. Infection with anaerobic and microaerophilic bacteria showed host specificity, where Fusobacter, Prevotella, Veillonella, Propionibacter, Gemella, and Helicobacter coexist in same patients. Coexistence of anaerobic bacteria was mainly limited to patients with pancreatic cancer. Clostridium and Peptoclostridium spp. were detected in $80 \%$ and $86 \%$ of the patients, where highest relative abundance rates were detected in patients with elevated ALP levels and leukocytosis, respectively. Patients with leukocytosis presented higher rates of colonization with Pseudomonas, Cronobacter, Enterococcus, Klebsiella, Peptoclostridium, and Streptocossus. H. pylori was detected in 4 patients with and 1 patient without CBD stone. Higher diversity in the bacterial population was detected in patients with CBD stone compared with those lacking the stone, as richness of an unclassified member of Alphaproteobacteria, plus Helicobacter, Enterobacter/ Cronobacter spp., Sphingomonas, Prevotella, Fusobacterium and Aeromonas were detected. Further studies are needed to show impact of these bacteria in human health or diseases through their interaction with host and virulence potential.

\section{Introduction}

Gallstone disease is one of the most common human gastrointestinal diseases with incidence rate between 11-15\% among adults [1]; [2]; [3]. The important role of gallstones in the development of gallbladder cancer was already documented [4]. Formation of gallstone is a complex process involving the interaction between the environmental and genetic factors [5]. No definite etiological risk factors have been described for formation of the gallstone; however, several factors, including the ethnicity, gender (Female vs male), age (advanced-age vs young people), high/low body mass index, dyslipidemia, diabetes mellitus, the metabolic syndrome, high-fat-diet, lifestyle factors (e.g. reduced physical activity), the socioeconomic status, underlying chronic diseases such as cystic fibrosis and Crohn's disease, and drugs (e.g. ceftriaxone) showed epidemiological links that are varied between different countries [6].

Most studies have already focused on the abnormal metabolism and secretion of bile acids and cholesterol as the primary pathophysiological defect in the gallstones formation [7]. Brown pigmented stones are formed in the common bile duct (CBD), which cause a type of biliary diseases, known as choledocholithiasis. It also can be formed in the intrahepatic bile ducts (hepatolithiasis) [6]. It is believed that CBD stones, especially brown pigment stones, are resulted from infection with bacteria and parasites/helminthes secondary to obstruction [8]. This type of stones could cause recurrent cholangitis and are correlated with primary biliary cirrhosis [9]. Although cholestasis by any etiology or changes in the antimicrobial composition of bile components predisposes patients to infection with these microbes, direct interaction of bacteria with this tissue or indirectly by the reactivity of their metabolites or enzymes (such as $\beta$ glucuronidase) could promote aggregation of cholesterol monohydrate crystals, which is a component of biliary sludge [9];[10]. Microbiota of upper digestive tract seems to play major role in this interplay. Several studies showed that these bacteria can reach themselves to the bile duct and the gallbladder in patients with underlying disease; however, few data exist about their composition and fate, either as transient or as common microbiota [11]. In previous studies, many bacteria, such as Klebsiella pneumoniae, Escherichia coli, and Enterococcus faecium, were isolated from bile samples through cultivation [12] or identified by polymerase chain reaction (PCR)-based methods in bile or gallstone samples [13]. New data indicated more complex population of bacteria in the gastrointestinal tract, which their possible contribution in biliary tract diseases is not negligible. It is now well accepted that the vast majority of microbial species are uncultivable and as a result culture-independent molecular methods are sought to characterize the causative agents of infectious diseases [14]. $16 \mathrm{~S}$ ribosomal gene-specific Next generation sequencing (16s rRNA-NGS) is a culture-independent technique used to identify the population and the proportion of bacterial species within an environment, including human biological samples, without any need for isolation or identification by traditional cultural methods [15]. This knowledgecan in turn be used to introduce the effective prevention and protection methods for infectious diseases[16]. Recently, the application of NGS technologies on biliary samples provided a more exhaustive understanding of the bacterial community within human biliary tract [17].

Improved understanding of the gallstone microbiota composition and their involvement in disease would be used as guide to administration of more efficient antibiotic regimens, which is in turn a great step toward personalized medicine. Unlike conventional methods for identifying bacteria, such as classical cultivations or PCR, NGS provides a comprehensive picture of the biliary tract microbiota [18]. Accordingly, the objective of the present study was $16 S$ rDNANGS analysis of bile samples in symptomatic patients with underlying diseases after endoscopic retrograde cholangiopancreatography. Association of the characterized bacteria with changes in liver function tests and markers of inflammation were also measured in this study.

\section{Material And Methods}

\subsection{Patients and sample collection.}

The study was performed at ERCP unit of Taleghani Hospital in Tehran, Iran. Suspected patients to choledocholithiasis who presented with jaundice and fever were enrolled. Bile samples were collected from the common bile ducts of 15 patients ( 9 men, 6 women) with mean age of $48.07 \pm 16.48$ years between August 2015 and October 2016. Clinical features and demographic data were filled for all patients through a questionnaire on the day of admission. Written informed consent was also obtained from all the patients under a protocol approved by the Ethical Review Committee of the Research Institute for Gastroenterology and Liver Diseases in Shahid Beheshti University of Medical Sciences.

\subsection{Culture and characterization of the bacterial isolates}

Nearly, $5 \mathrm{ml}$ of bile samples were collected from the patients in sterile tubes at the time of the examination. Culture on Blood agar and MacConkey agar media was done aerobically at $37^{\circ} \mathrm{C}$ for 24 hours. Biochemical characterization was done according to the Bergey's Manual of Systematic Bacteriology [19].

Page $2 / 10$ 


\subsection{DNA extraction from bile samples}

Bile samples were transported to the laboratory in sterile containers immediately after collection and were subsequently frozen at $-20^{\circ} \mathrm{C}$ for future analysis. Total DNA was extracted by phenol chloroform method as previously described by Vu-Thien et al. [20, 21]. The extracted DNA samples were suspended into Tris-EDTA buffer and used as a template for amplification with specific primers (27f mix-forward: AGMGTTYGATYMTGGCTCAG and R: 5'GCTGCCTCCCGTAGGAGT-3') [22]. The quality and quantity of DNA extracts was measured by electrophoresis on $1.2 \%$ agarose gel and also by using Nanodrop, respectively )Labtech, Palaiseau, France).

\subsection{S rDNA sequencing, OTU analysis, alignment, and classification}

Library preparation and subsequent paired end sequencing was conducted according to $16 S$ rDNA sequencing service offered by BGI Genomics Co, China. Mothur pipeline was used for the downstream analyses. Accordingly, reverse and forward reads were combined into contigs using the "make.contigs "command. Subsequently data cleaning was carried out on the resulted contigs employing the "screen.seqs" command. "unique.seqs" command was then used to remove duplicate sequences and the "count.table" of unique sequences was generated applying "count.seqs" command. Afterwards unique sequences were aligned on Silva-V4 reference fasta file followed by running "screen.seqs" to remove poorly aligned reads. After pre-clustering of reads, chimeric sequences resulted from PCR amplification step were removed from the sequence data using "chimera.vsearch" function. In order to remove nonbacterial sequences "classify.seqs" and "remove.lineage" commands were subsequently applied. OTU picking was carried out using "cluster.split" command based on $97 \%$ sequence similarity and taxonomic assignment was determined by "classify.otu" command.

To specify number of sequences in each sample "count.groups" was used and then alpha diversity rarefaction curve was generated. Beta diversity was also calculated among 15 samples and its result was visualized in the form of heatmap. For validation purpose, KRAKEN metagenomics pipeline was also employed. FastQ files were feed in to Galaxy bioinformatics pipeline and the following steps were taken to characterize the bacterial composition of each sample. FastQ files were first trimmed using Trimmomatic program $[23,24]$ to remove low quality bases from both ends of every read. The trimmed paired files for each sample were then introduced into Kraken taxonomic sequencing classifier [25, 26], a sensitive and highly precise algorithm, choosing "Bacteria" as the database used by Kraken. In brief, Kraken searches for k-mers within each read in a database of k-mers and tries to assign the lowest common ancestor (LCA) to each k-mer in a taxonomic tree for all genomes that contain that k-mer. A single taxonomic label is subsequently assigned to each read following analyzing the set of LCA that corresponds to all k-mers constituting each read. Kraken report program was then used to get the number of reads covered by the clade rooted at each specified taxon and also number of reads assigned directly to this taxon.

\section{Results}

\subsection{Patients and infection}

CBD stone was detected in $60 \%$ (9/15) of the suspected patients, while other complications were included jaundice (2/15), epigastric pain (2/15), and fever of unknown origin (1/15). Hydatid cyst was reported in one patient with fever. All, except two patients presented past medical history, included diabetes mellitus (3/15), pancreatic cancer (3/15), incomplete cholecystectomy (2/15), sphincterotomy (1/15), chronic kidney disease (1/15), ischaemic heart disease (1/15), and hypertension (3/15). Based on the culture method, infection with Pseudomonas aeruginosa and E. coli was detected in 26.66\% (4/15) and 13.3\%, (2/15) of the patients, respectively (Table 1). 
Table 1

Diversity of cultivable bacteria in bile samples of patients with distinct types of underlying diseases after endoscopic retrograde cholangiography.

\begin{tabular}{|c|c|c|c|c|c|c|c|c|c|c|}
\hline \multirow{2}{*}{$\begin{array}{l}\text { Patients } \\
\text { Number }\end{array}$} & \multirow{2}{*}{$\begin{array}{l}\text { AST } \\
\text { IU/La. }\end{array}$} & \multirow{2}{*}{$\begin{array}{l}\text { ALT } \\
\text { IU/L }\end{array}$} & \multirow[t]{2}{*}{ ALPIU/L } & \multirow{2}{*}{$\begin{array}{l}\text { WBC } \\
\text { per } \mu \text { l }\end{array}$} & \multirow{2}{*}{$\begin{array}{l}\text { BUN } \\
\mathrm{mg} / \mathrm{dl}\end{array}$} & \multirow{2}{*}{$\begin{array}{l}\mathrm{CR} \\
\mathrm{mg} / \mathrm{dl}\end{array}$} & \multirow[t]{2}{*}{ Diseases } & \multicolumn{3}{|c|}{ Culture and Medical history } \\
\hline & & & & & & & & $\begin{array}{l}\text { Aerobic } \\
\text { culture }\end{array}$ & $\begin{array}{l}\text { Anaerobic } \\
\text { culture }\end{array}$ & Past Medical History \\
\hline 1 & 25 & 30 & 400 & 7600 & 14 & 1 & CBD stone & Negative & Negative & $\begin{array}{l}\text { Ischaemic heart } \\
\text { disease }\end{array}$ \\
\hline 2 & 294 & 560 & 1401 & 6700 & 22 & 0.8 & CBD stone & Negative & Negative & Diabetes mellitus \\
\hline 3 & 75 & 62 & 902 & 7600 & 32 & 2.4 & Jaundice & Pseudomonas & Negative & Not reported \\
\hline 4 & 17 & 15 & 757 & 7200 & 21 & 1.3 & CBD stone & Negative & Negative & Diabetes mellitus \\
\hline 5 & 12 & 40 & 150 & 5800 & 10 & 1 & CBD stone & Negative & Negative & $\begin{array}{l}\text { Hypertension, } \\
\text { Diabetes mellitus }\end{array}$ \\
\hline 6 & 12 & 40 & 280 & 5200 & 25 & 0.9 & Epigastric pain & Negative & Negative & Pancreatic cancer \\
\hline 7 & 14 & 45 & 350 & 8600 & 23 & 0.9 & CBD stone & Negative & Negative & Cholecystectomy \\
\hline 8 & 16 & 46 & 450 & 7800 & 24 & 0.8 & CBD stone & Pseudomonas & Negative & $\begin{array}{l}\text { Chronic kidney } \\
\text { disease }\end{array}$ \\
\hline 9 & 13 & 42 & 340 & 5600 & 24 & 1 & $\begin{array}{l}\text { Fever, Hydatid } \\
\text { cyst }\end{array}$ & Negative & Negative & Hypertension \\
\hline 10 & 182 & 147 & 808 & 8400 & 13 & 0.8 & $\begin{array}{l}\text { Fever of unknown } \\
\text { origin }\end{array}$ & E. coli & Negative & $\begin{array}{l}\text { Previous } \\
\text { sphincterotomy }\end{array}$ \\
\hline 11 & 50 & 18 & 357 & 41700 & 19 & 0.7 & Jaundice & E. coli & Negative & Not reported \\
\hline 12 & 45 & 58 & 2585 & 16700 & 14 & 0.9 & Epigastric pain & Negative & Negative & Pancreatic cancer \\
\hline 13 & 17 & 28 & 615 & 6300 & 11 & 0.7 & CBD stone & Negative & Negative & Hypertension \\
\hline 14 & 323 & 255 & 412 & 11500 & 17 & 0.8 & CBD stone & Pseudomonas & Negative & Cholecystectomy \\
\hline 15 & 22 & 16 & 768 & 25000 & 15 & 0.8 & CBD stone & Pseudomonas & Negative & Hypertension \\
\hline
\end{tabular}

\section{2. $16 S$ rDNA sequencing}

Out of 14.4-55.5 Mbp raw data, 402075 pairs of reads were recovered following the $16 S$ rDNA-NGS sequencing after trimming, filtering and binning (mean length: 300 bp), ranging from 13911 to 47952 sequences per sample (Average 26805 tags). OTU assignment with Mothur showed a total of 181 OTUs with an OTU coverage of $99.9 \% \pm 0.05 \%$ (mean \pm S. D) (Supplementary Fig. 3). Dominant phyla across these samples, as was determined by Kraken, were included Proteobacteria (100\%, 15/15), Firmicutes (100\%, 15/15), Actinobacteria (100\%, 15/15), Bacteroidetes (93.3\%, 14/15), Cyanobacteria/Chloroplast (73.3\%, 11/15), Fusobacteria (73.3\%, 11/15), Acidobacteria (46.6\%, 7/15), and Deinococcus-Thermus (26.6\%, 4/15) (Fig. 1).

Statistical analysis showed no correlation among total numbers of the reads for bacterial phyla and ALP levels and WBC counts in the patients. Moreover, no significant difference was detected between the presence and numbers of reads between patients with CBD stone and those with no stone. Proteobacteria (mean \pm SD of $23877.3 \pm 13197$ ) and Firmicutes (3427.7 \pm 4803 ) were among the most frequent phyla in the studied bile samples (Table 2). While patients with diabetes and CBD stone showed highest amounts of Firmicutes (CBD vs non-CBD stone, mean No. of reads \pm SD of $4064 \pm 5932 v s 2364+2366$, respectively), the highest count of Proteobacteria was measured in a patient with sphincterotomy. Pseudomonas spp. constituted highest population of the bacterial genera in patients with a history of cholecystectomy, sphincterotomy, pancreatic cancer, and hydatid cyst. Pseudomonas spp., Achromobacter, and Klebsiella in patient with sphincterotomy, Pseudomonas + Stenothrophomonas together with Enterococcus and Klebsiella in patients with pancreatic cancer, and Pseudomonas together with Cronobacter, Enterobacter, and Caulobacter in patients with cholecytectomy were among dominant genera with highest number of reads in the studied samples. Bacterial genera that their presences were confirmed in all the bile samples were included Cronobacter, Pseudomonas, Enterococcus, Achromobacter, Klebsiella, and Enterobacter spp. The most common bacteria were as follows: Cronobacter, Pseudomonas, Escherichia/Shigella, Enterococcus, Sphingomonas, Achromobacter, Klebsiella, Morganella, Stenotrophomonas, Streptococcus, Brevundimonas, Lactobacillus, Burkhuldria, and Clostridium spp. Infection with anaerobic and microaerophilic bacteria showed host specificity, where Fusobacter, Prevotella, Veillonella, Propionibacter, Gemella, and Helicobacter were detected together in same patients. The coexistence of anaerobic bacteria, mainly included Clostridium, Fusobacters, Veillonella, Propionibacterium, Peptoclostridium, Tannerella, and Prevotella in a sample was mainly detected in patients with pancreatic cancer (P6, P3, and P15); however, this presence was also detected in patients with CBD stone (P13) and a patient with sphincterotomy (P10). Clostridium and Peptoclostridium spp. were detected in $80 \%$ and $86 \%$ of the patients, where highest relative abundance rates were detected in patients with elevated ALP levels and WBC counts, respectively. In patients with elevated WBC counts, higher rates of colonization with Pseudomonas, Cronobacter, Enterococcus, Klebsiella, Peptoclostridium, and Streptocossus were measured. H. pylori was detected in 4 patients with CBD stone $(4 / 9,44.4 \%)$ and 1 patient with no CBD stone (1/6, $16.6 \%)$. 
Table 2

Number of paired reads for the characterized bacterial phyla in bile samples of patients with biliary diseases.

\begin{tabular}{|c|c|c|c|c|c|c|c|c|c|c|c|c|}
\hline & P1 & P2 & P3 & P4 & P5 & P6 & P7 & P8 & P9 & P10 & P11 & P1 \\
\hline WBC count & Normal & Normal & Normal & Normal & Normal & Normal & Normal & Normal & Normal & Normal & High & $\mathrm{Hi}$ \\
\hline ALP & Moderate & $\begin{array}{l}\text { Very } \\
\text { high }\end{array}$ & High & High & Normal & Moderate & Moderate & Moderate & Moderate & Moderate & Moderate & $\begin{array}{l}\text { Dr } \\
\text { hic }\end{array}$ \\
\hline CBD Stone & CBD & CBD & No & CBD & CBD & No & CBD & CBD & No & No & No & Nc \\
\hline Proteobacteria & 12498 & 25100 & 18181 & 3229 & 37149 & 13546 & 30128 & 10832 & 42912 & 47690 & 27902 & 31 \\
\hline Firmicutes & 1023 & 11977 & 1505 & 16390 & 350 & 764 & 1 & 508 & 3803 & 255 & 6737 & 14 \\
\hline Bacteroidetes & 810 & 6 & 224 & 47 & 35 & 501 & 1 & 1781 & 0 & 2 & 1 & 12 \\
\hline Cyanobacteria & 179 & 1 & 24 & 3 & 0 & 46 & 0 & 26 & 4 & 0 & 1 & 0 \\
\hline Actinobacteria & 146 & 2 & 168 & 6 & 1 & 158 & 2 & 287 & 6 & 1 & 64 & 15 \\
\hline Fusobacteria & 38 & 7586 & 3 & 2 & 0 & 9 & 0 & 77 & 1 & 0 & 1 & 0 \\
\hline Acidobacteria & 20 & 0 & 0 & 2 & 0 & 4 & 1 & 104 & 0 & 0 & 0 & 0 \\
\hline $\begin{array}{l}\text { Deinococcus- } \\
\text { Thermus }\end{array}$ & 12 & 0 & 0 & 2 & 0 & 0 & 0 & 68 & 0 & 0 & 0 & 0 \\
\hline $\begin{array}{l}\text { Candidatus } \\
\text { Saccharibacteria }\end{array}$ & 9 & 0 & 0 & 0 & 0 & 0 & 0 & 0 & 0 & 0 & 0 & 0 \\
\hline Spirochaetes & 0 & 0 & 0 & 0 & 0 & 0 & 0 & 0 & 0 & 0 & 0 & 0 \\
\hline Tenericutes & 0 & 0 & 0 & 0 & 0 & 0 & 0 & 0 & 0 & 0 & 0 & 0 \\
\hline Spirochaetes & 0 & 0 & 0 & 0 & 0 & 0 & 0 & 0 & 0 & 0 & 0 & 0 \\
\hline
\end{tabular}

WBC, White blood cell; ALP, Alkaline phosphatase; CBD, Common bile duct stone; No, no stone in common bile duct.

Analysis of OTUs at species levels showed dominance of the following species with > 100 reads per sample: P. putida (12/15), S. maltophilia (11/15), Pseudomonas aeruginosa (10/15), Pseudomonas stuatzeri group (6/15), Achromobacter xylosoxidans (6/15), Acinetobacter calcoaceticus/ baumanii complex (5/15), Chitinophagapinesis (4/15), Enterococcus hirae (4/15), Sphingomonas wittich (4/15), Burkhulderia genera incertae (3/15), E. casseliflavus (2/15), Streptococcus anginosus (2/15), Fusobacterium nucleatum (2/15), Haemophilus parasuis (2/15), H. parainfluenzae (2/15), Veillonella paravula (2/15), Alkalilimnicolaehrlichii (2/15), Prevotella melaninogenica (2/15), Sphingopyxis alaskensis (1/15), Aeromonas verinii (1/15), Providencia stuartii (1/15), Brevundimonas subvibrioides (1/15), Rothia denticariosa (1/15), Novosphingobium aromaticivorans (1/15), Serratia marcescens (1/15), and K. pneumoniae (1/15).

\subsection{Effects on Richness and Diversity}

Enrichment of some of the bacterial genera in patients with CBD stone compared with non-CBD stone were detected based on the measured relative abundance rates. Accordingly, based on the Kraken results, Helicobacter, and Enterobacter/ Cronobacter spp. were among the more frequent genera that observed in patients with CBD stone. While mean values of the relative abundance rates didn't show significant difference, variances of the two bacterial genera were significantly different ( $p$ value $<0.0001$, Supplementary Figs. 1 and 2 ). Similarly, this difference was also confirmed in the cases of Sphingomonas ( $p$ value $=0.0033)$, Prevotella $(p$ value $=0.009)$, Fusobacterium $(p$ value $<0.0001)$ and Aeromonas $(p$ value $<0.0001)$. Klebsiella, Stenotrophomonas and Achromobacter spp. showed higher relative abundance rates in patients with no CBD stone $(p$ value $<0.0001$ and 0.0007 , respectively). Results of Mothur showed richness of an unclassified member of Alphaproteobacteria, Helicobacter, Prevotella, Fusobacterium, and Aeromonas, in patients with $\mathrm{CBD}$ stone, while unclassified members of Pseudomonadceae, Enterobacteriaceae, and Gammaproteobacteria were enriched in the non-CBD stone group (Table 3). The bacterial taxa showed higher diversity in patients with CBD stone (158/181, $87.3 \%)$, compared with non-CBD stone patients (120/181, 66.3\%). 
Table 3

Differences in relative abundance of main bacterial OTUs calculated using Mothur in patients with CBD stone and those with no CBD stone.

\begin{tabular}{|c|c|c|c|c|}
\hline & \multicolumn{4}{|l|}{ Relative abundance (\%) } \\
\hline & CBD stone (mean $\pm \mathrm{STD})$ & $\begin{array}{l}\text { Non-CBD stone } \\
\text { (mean } \pm \text { STD) }\end{array}$ & $\begin{array}{l}\text { T test } \\
\text { ( } p \text { value) }\end{array}$ & $\begin{array}{l}\mathrm{F} \text { test } \\
\text { ( } p \text { value) }\end{array}$ \\
\hline Pseudomonadaceae_unclassified & $3.23 \pm 4.2$ & $8.27 \pm 7.5$ & NS & NS \\
\hline Alphaproteobacteria_unclassified & $3.13 \pm 4.94$ & $0.26 \pm 0.42$ & 0.18 & $<0.0001$ \\
\hline Enterobacteriaceae_unclassified & $8.81 \pm 8$ & $15.7 \pm 10.9$ & NS & NS \\
\hline Gammaproteobacteria_unclassified & $2.62 \pm 3.12$ & $8 \pm 7.67$ & 0.08 & 0.026 \\
\hline Helicobacter & $2.1 \pm 3.6$ & $0.8 \pm 1.48$ & NS & NS \\
\hline Prevotella & $1.6 \pm 2.47$ & $0.52 \pm 1.27$ & NS & NS \\
\hline Fusobacterium & $1.75 \pm 1.78$ & $0.32 \pm 0.5$ & 0.083 & 0.013 \\
\hline Aeromonas & $1.26 \pm 1.86$ & $0.33 \pm 0.5$ & 0.26 & 0.01 \\
\hline
\end{tabular}

\section{Discussion}

It is assumed that a combination of risk factors, including genetic background, anatomical, physiological, and immunological disorders, history of medications, and composition of the gut microbiota could mediate distinct types of diseases in the biliary system. Ability of microbes for interaction with the biliary tract, production of toxins, metabolites, enzymes, possibly play roles in promotion of these disorders. Recent progresses in the development of advance technologies for DNA sequencing has provided us the opportunity to better analyze common members of the microbiome in association with this system, and study of their involvement in related disorders. The primary purpose of this study was to analyze composition of the bile microbiota in patients with biliary tract diseases using $16 S$ rDNA sequencing and their correlation with CBD stone formation, the elevated WBC count and ALP level., were investigated. Accordingly, high level of diversity in the composition of the bile microbiome was revealed in the studied samples. While this analysis confirmed the existence of $P$. aeruginosa as one of the most prevalent bacteria in the biliary tract, which was also in accordance to the results of culture-based method, most of the cases showed mixed type infection by variety of aerobic and anaerobic bacteria. The results showed no statistically significant difference in the composition of the bacteria phyla and genera between the patients with and without CBD stone. The most common phyla in our samples were included, Proteobacteria, Firmicutes, Bacteroidetes, Actinobacteria; Cyanobacteria, and Fusobacteria, respectively. In a study by Pereira P. et al., Firmicutes, Bacteroidetes,

Proteobacteria, Fusobacteria, and Actinobacteria were orderly recorded as most common bacterial phyla in bile samples of patients with elevated serum ALP and patients with primary sclerosing cholangitis [27]. In a study by Ye F. et al., all of these phyla plus Synergistetes and TM7 were characterized in bile samples of patients with choledocholithiasis [28].Existence of Cyanobacteria, as was shown in our study, was similarly reported in a study on bile samples of patients with gallbladder stones [17]. Regardless to the status of CBD stone formation and underlying diseases in the studied patients, more frequent genera of the bacteria in the bile samples were included Cronobacter, Pseudomonas, Chitinophaga, Enterococcus, Sphingomonas, Achromobacter, Klebsiella, Aeromonas, Providencia, Stenotrophomonas, Synechococcus, Bradyrhizobium, Acinetobacter, Enterobacter, Lactococcus, Methylobacterium, Bacillus, Propionibacterium, Peptoclostridium, Alkalilimnicola, and Streptococcus. These genera showed completely different patterns in comparison to those characterized by Pereira P. et al. and Ye F. et al., where Prevotella, Streptococcus, Veillonella, Fusobacteriun, Haemophilus, Neisseria [27]), and E. coli and Klebsiella, Pyramidobacter, Enterococcus, Fusobacterium, Streptococcus, and Veillonella were dominant, respectively [28]. Although our results didn't show significant difference in the characterized bacterial genera in the bile samples of patients with and without bile duct stone, this association for gallstone was shown in the study of Wu et al. They showed this association for Lactococcus, Bacteroides, Caulobacter, Propionibacterium, Clostridium, Staphylococcus, Acinetobacter, Anoxybacillus, Arthrobacter, Chryseobacterium, and E. coli [17]. The presence of some bacterial genera in the studied bile samples, such as those that are linked with the oral and upper respiratory tract (e.g. Veillonella, Corynebacterium, Staphylococcus, Prevotella, Haemophilus, Neisseria, Legionella, Bordetella, Pseudomonas, and Prevotella), those colonizing the gastric tissue (Helicobacter, Lactobacillus, Leptotrichia, and Streptococcus), beside important enteric pathogens (e.g. Yersinia, Salmonella, C. difficile, C. perfringens), proposed this system as a suitable niche for their colonization and possibly the pathogenesis. Our results showed preference of Cronobacter, Pseudomonas, and Enterococcus for colonization and proliferation in the biliary tract, since higher numbers of sequence reads were detected. Cronobacter sakazakii could produce exopolysaccharide, which form biofilm and also encodes active efflux pumps that mediate resistance to bile salts [29]. These properties in A. baumanii could also mediate resistance to bile salts [30]. Ability of $C$. sakazakii for tolerance to $5 \%$ bile salts was shown in a study by MdFakruddin et al. [31]. In the case of Enterococcus, a permease protein (GltK), which is a glutamate/aspartate transport system, is responsible for its resistance to bile salts. Increased level of adherence ability of Enterococcus in the presence of bile to bile drain materials was established in a study by Waar K. et al., which could show its capacity of adaptation for site specific colonization in this tract [32]. In the case of Salmonella, upregulation of genes, including RpoS-dependent and independent stress response genes, lipopolysaccharide, exoplolysacharide [33], the outer membrane, the cytoplasmic membrane, efflux pump systems, genes of the multiple antibiotic resistance (mar) and PhoPQ regulons, and those mediating in DNA repair functions, play crucial role in bile resistance [34]. Resistance property of these bacteria to bile salts could facilitate their persistent residence, promotion of the infection and pathogenesis, stone formation through precipitation of bile salts, induction of inflammatory response, or DNA damage. Some of the bacteria could deconjugate bilirubin, leading to their precipitation as calcium salts. Deconjugation of bile salts could cause by bacterial bile salt hydrolases, which their presence in Lactobacillus, Enteroccocus, Bifidobacterium, Bacteroides, Methanobrevibacter, Methanosphera, and Clostridium were already established [35]. Oxidation and epimerization of bile salts, which generates isobile ( $\beta$-hidroxy) salts, is also mediated by some of the enteric bacteria that was characterized in our studied bile samples, including Clostridium, Peptostreptococcus, Bacteroides, Eubacterium, and Escherichia coli [36]. 
Ability of bacteria for secretion of enzymes, such as $\beta$-glucuronidase, phospholipases, bile acid hydrolases [17], and urease are involved in gallstone formation and calcium salts precipitation [17] [37]]. In our study, we characterized urease producing bacteria, including Clostridium, Corynebacterium, Citrobacter, Enterobacter, Klebsiella, Morganella, Proteus, Providencia, Haemophilus, Staphylococcus, Streptococcus, Yersinia, Pseudomonas, and Helicobacter in bile samples of the patients; however, no significant difference in their relative abundance was detected, except for Enterobacter (> 8 fold) and Helicobacter (> 240 fold) that showed higher mean values in patients with CBD stone. There are contrary reports about involvement of Helicobacter spp. in biliary tract diseases. In a study by Jahani Sherafat S. et al., Helicobacter pylori was detected in $2.7 \%$ of patients with gallstone that was lower than those reported before by Farshad Sh. et al. (18.1\%), Monstein HJ et al. (55.5\%), and Fatemi SM. et al (14.3\%) [38] [39] [40]. Association of H. pylori and non-pylori Helicobacter spp., such as $H$. hepaticus, H. pylori, H. bilis, with increased risk of biliary tract cancers (Cholangiocarcinoma) was previously reported [41]. This involvement was not limited to gallstone disease, but also confirmed among patients with hepatolithiasis (53\%), and those with choledocholithiasis (47\%) [42]. Interestingly, in our study patients with $H$. pylori infection (P1, P8, P13, P15, P6) showed more complex microbiome compared with $H$. pylori negative patients. This finding was supported by other studies in humans or animals, where coinfection with several bacteria, including Helicobacter spp., was determined as a prerequisite for induction of cholesterol gallstones [17]; [37]. It is not clear yet that how urease can promote gallstone formation in cooperation with other bacteria. In animal models it was shown that coinfection with several bacteria could develop cholesterol gallstones at a higher level than the infected cases with single bacterial genus (80\% vs $40 \%)$ [17].

$\beta$-glucuronidase, the other bacterial enzyme that is linked with gallbladder and CBD stone formation is encoded by Bacteroides, Clostridium perfringens, some members of Enterobacteriaceae (e.g. Escherichia coli), Firmicutes (e.g. Lactobacillus, Streptococcus, Enterococcus, Clostridium, Ruminococcus, Roseburia, and Faecalibacterium), a species of Actinobacteria (Bifidobacterium dentium), Anaerococcus prevotii, and Desulfitobacterium hafniense [43, 44]. In our study, among the noted bacteria, the highest amounts of sequence read in patients with CBD stone were related to Bacteroides, C. perfringens, Lactobacillus, Streptococcus, Enterococcus, Clostridium spp., and Bifidobacterium; however, they were also presented in patients with non-CBD stone in lower abundances.

Our results at the species level showed the existence of Sphingomonas wittichii, Stenotrophomonas maltophilia group, Chitinophaga pinensis, Providencia stuartii, together with Pseudomonas putida group, Pseudomonas stutzeri subgroup, Pseudomonas aeruginosa group, Enterococcus hirae, Achromobacter xylosoxidans, Brevundimonas subvibrioides, Acinetobacter calcoaceticus/baumannii complex, Veillonella parvula, and Prevotella melaninogenica in amounts $>100$ reads per sample. High counts of these bacteria showed their ability for colonization, which promote their probable interaction with the biliary system. In the case of $S$. wittichii, bioinformatic analysis showed that its genome contains several major virulence factors resembling those characterized in Pseudomonas, Legionella, Brucella, and Bordetella spp; however, its involvement in human infection has not been established yet [45]. Further studies are needed to determine mechanisms that are used by these bacteria for tolerating bile salts and to characterize virulence factors promoting their pathogenicity.

There are contrary reports about impact of underlying diseases on the composition of the bile microbiota [27, 46, 47]. In our study, dominance of few bacterial genera and lower diversity was detected in patients who subjected to cholecystectomy (P14, P7) and those with history of sphincterotomy (P10). In a study by Liang T. et al., it was determined that patients with sphincter of Oddi laxity had more severe bacterial infection and stronger lithogenicity [46]. Further studies on greater numbers of patients are needed to show this effect.

Comparison of analysis methods by Mothor and Kraken showed a congruency between the results. Enrichment of an unclassified member of Alphaproteobacteria, which was characterized by Mothur, was in accordance with higher abundance of Sphingomonas, (A member of this phylum that was determined by the Kraken) in patients with CBD stone. Similarly, dominance of unclassified members of Enterobacteriacea and Pseudomonadaceae, as was detected by Mothur, in non-CBD stone samples were in congruence with the abundance of Klebsiella and Pseudomonas spp. that were detected by Kraken in non-CBD stone group, respectively. Despite this similarity, Kraken showed a preference for detection of higher numbers of OTUs, genera of the bacteria (202 vs 181 genera), and ability for characterization of some of the sequences at species level.

In general, $16 S$ rDNA analysis showed high level of diversity in the composition of the bile microbiome, which was mainly included uncultivable and fastidious bacteria. Higher relative abundance rates of some bacterial taxa in association to formation of CBD stone and elevated ALP and WBC levels was detected in the patients; however, presence of these bacteria showed no statistically significant difference $v s$ those with no-CBD stone. These results showed impact of underlying diseases, such as cholecystectomy, sphincterotomy, and pancreatic cancer on the bacterial composition of the biliary tract; however, performing this experiment with the larger number of patients will help to suggest actual correlation between the presence of specific bacterial communities and disease phenotype.

\section{Declarations}

\section{Acknowledgments}

The authors thank staff of endoscopic retrograde cholangiography unit of Ayatollah Taleghani hospital for their cooperation in this study. All authors have read the journal's authorship agreement and the final manuscript has been approved by all named authors. This project is supported by a grant from Research Institute for Gastroenterology and Liver Diseases, Shahid Beheshti University of Medical Sciences, Tehran, Iran.

\section{Conflict of interest}

All the authors declared no competing interests.

\section{References}


1. Everhart JE KM, Hill M, Maurer KR. Prevalence and ethnic differences in gallbladder disease in the United States. Gastroenterology. 1999,117(3):632-9, doi: 10.1016/s0016-5085(99)70456-7.

2. Sandler RS, Everhart JE, Donowitz M, Adams E, Cronin K, Goodman C, et al. The burden of selected digestive diseases in the United States. Gastroenterology. 2002,122(5):1500-11, doi: 10.1053/gast.2002.32978.

3. Bansal A, Akhtar M, Bansal AK. A clinical study: prevalence and management of cholelithiasis. 2016. 2016,1(3):6.

4. Tsuchiya Y, Loza E, Villa-Gomez G, Trujillo CC, Baez S, Asai T, et al. Metagenomics of Microbial Communities in Gallbladder Bile from Patients with Gallbladder Cancer or Cholelithiasis. Asian Pac J Cancer Prev. 2018. doi: 10.22034/APJCP.2018.19.4.961.

5. Portincasa P, Moschetta A, Palasciano G. Cholesterol gallstone disease. The Lancet. 2006,368(9531):230-9, doi: 10.1016/S0140-6736(06)69044-2.

6. Stinton LM, Shaffer EA. Epidemiology of gallbladder disease: cholelithiasis and cancer. Gut Liver. 2012,6(2):172-87, doi: 10.5009/gnl.2012.6.2.172.

7. Wang DQH, Cohen DE, Carey MC. Biliary lipids and cholesterol gallstone disease. J Lipid Res. 2009,50 Suppl(Suppl):S406-S11, doi: 10.1194/jlr.R800075JLR200.

8. Vítek L, Carey MC. New pathophysiological concepts underlying pathogenesis of pigment gallstones. Clin Res Hepatol Gastroenterol. 2012,36(2):122-9, doi: 10.1016/j.clinre.2011.08.010.

9. Reshetnyak VI. Concept of the pathogenesis and treatment of cholelithiasis. World J Hepatol. 2012,4(2):18-34, doi: 10.4254/wjh.v4.i2.18.

10. Wang HH, Portincasa P, Liu M, Tso P, Wang DQH. Similarities and differences between biliary sludge and microlithiasis: Their clinical and pathophysiological significances. Liver Research. 2018,2(4):186-99, doi: https://doi.org/10.1016/j.livres.2018.10.001.

11. Petrov VA, Fernández-Peralbo MA, Derks R, Knyazeva EM, Merzlikin NV, Sazonov AE, et al. Biliary microbiota and bile acids composition in cholelithiasis. bioRxiv. 2018:469825, doi: 10.1101/469825.

12. Schneider J, De Waha P, Hapfelmeier A, Feihl S, Römmler F, Schlag C, et al. Risk factors for increased antimicrobial resistance: a retrospective analysis of 309 acute cholangitis episodes. Journal of Antimicrobial Chemotherapy. 2013,69(2):519-25, doi: 10.1093/jac/dkt373.

13. Swidsinski A, Khilkin M, Pahlig H, Swidsinski S, Priem F. Time dependent changes in the concentration and type of bacterial sequences found in cholesterol gallstones. Hepatology. 1998,27(3):662-5, doi: 10.1002/hep.510270304.

14. Cavaletti L, Monciardini P, Bamonte R, Schumann P, Rohde M, Sosio M, et al. New lineage of filamentous, spore-forming, gram-positive bacteria from soil. Appl Environ Microbiol. 2006,72(6):4360-9, doi: 10.1128/AEM.00132-06.

15. Salipante SJ, Sengupta DJ, Rosenthal C, Costa G, Spangler J, Sims EH, et al. Rapid 16S rRNA Next-Generation Sequencing of Polymicrobial Clinical Samples for Diagnosis of Complex Bacterial Infections. PLOS ONE. 2013,8(5):e65226, doi: 10.1371/journal.pone.0065226.

16. Mendes LW, Tsai SM. Distinct taxonomic and functional composition of soil microbiomes along the gradient forest-restinga-mangrove in southeastern Brazil. Antonie van Leeuwenhoek. 2018,111(1):101-14, doi: 10.1007/s10482-017-0931-6.

17. Wu T, Zhang Z, Liu B, Hou D, Liang Y, Zhang J, et al. Gut microbiota dysbiosis and bacterial community assembly associated with cholesterol gallstones in large-scale study. BMC Genomics. 2013,14(1):669, doi: 10.1186/1471-2164-14-669.

18. Shen H, Ye F, Xie L, Yang J, Li Z, Xu P, et al. Metagenomic sequencing of bile from gallstone patients to identify different microbial community patterns and novel biliary bacteria. Scientific Reports. 2015,5:17450, doi: 10.1038/srep17450. https://www.nature.com/articles/srep17450\#supplementaryinformation.

19. Holt JG, Williams ST, Holt. Bergey's manual of systematic bacteriology, Vol. 4. Lippincott Williams \& Wilkins, 1989.

20. Sambrook J, Russell DW. Purification of nucleic acids by extraction with phenol: chloroform. Cold Spring Harbor Protocols. 2006,2006(1):pdb. prot4455.

21. Vu-Thien H, Corbineau G, Hormigos K, Fauroux B, Corvol H, Clément A, et al. Multiple-Locus Variable-Number Tandem-Repeat Analysis for Longitudinal Survey of Sources of \&lt,em\&gt,Pseudomonas aeruginosa\&lt,/em\&gt, Infection in Cystic Fibrosis Patients. Journal of Clinical Microbiology. 2007,45(10):3175, doi: 10.1128/JCM.00702-07.

22. Walker AW, Martin JC, Scott P, Parkhill J, Flint HJ, Scott KP. 16S rRNA gene-based profiling of the human infant gut microbiota is strongly influenced by sample processing and PCR primer choice. Microbiome. 2015,3:26-, doi: 10.1186/s40168-015-0087-4.

23. Bolger AM, Lohse M, Usadel B. Trimmomatic: a flexible trimmer for Illumina sequence data. Bioinformatics. 2014,30(15):2114-20.

24. Bolger AM, Lohse M, Usadel B. Trimmomatic: a flexible trimmer for Illumina sequence data. Bioinformatics. 2014,30(15):2114-20, doi: 10.1093/bioinformatics/btu170.

25. Valenzuela-González F, Martínez-Porchas M, Villalpando-Canchola E, Vargas-Albores F. Studying long 16S rDNA sequences with ultrafast-metagenomic sequence classification using exact alignments (Kraken). Journal of microbiological methods. 2016,122:38-42.

26. Neves ALA, Li F, Ghoshal B, McAllister T, Guan LL. Enhancing the Resolution of Rumen Microbial Classification from Metatranscriptomic Data Using Kraken and Mothur. Front Microbiol. 2017,8:2445-, doi: 10.3389/fmicb.2017.02445.

27. Pereira P, Aho V, Arola J, Boyd S, Jokelainen K, Paulin L, et al. Bile microbiota in primary sclerosing cholangitis: Impact on disease progression and development of biliary dysplasia. PloS one. 2017,12(8):e0182924-e, doi: 10.1371/journal.pone.0182924.

28. Ye F, Shen H, Li Z, Meng F, Li L, Yang J, et al. Influence of the Biliary System on Biliary Bacteria Revealed by Bacterial Communities of the Human Biliary and Upper Digestive Tracts. PloS one. 2016,11(3):e0150519-e, doi: 10.1371/journal.pone.0150519.

29. Healy B, Cooney S, O'Brien S, Iversen C, Whyte P, Nally J, et al. Cronobacter (Enterobacter sakazakii): An Opportunistic Foodborne Pathogen. Foodborne Pathogens and Disease. 2009,7(4):339-50, doi: 10.1089/fpd.2009.0379.

30. López M, Blasco L, Gato E, Perez A, Fernández-Garcia L, Martínez-Martinez L, et al. Response to Bile Salts in Clinical Strains of Acinetobacter baumannii Lacking the AdeABC Efflux Pump: Virulence Associated with Quorum Sensing. Front Cell Infect Microbiol. 2017,7:143-, doi: 10.3389/fcimb.2017.00143.

Page $8 / 10$ 
31. Fakruddin M, Rahaman M, Ahmed MM, Hoque MM. Stress tolerant virulent strains of Cronobacter sakazakii from food. Biol Res. 2014,47(1):63-, doi: 10.1186/0717-6287-47-63.

32. Waar K, van der Mei HC, Harmsen HJM, Degener JE, Busscher HJ. Adhesion to bile drain materials and physicochemical surface properties of Enterococcus faecalis strains grown in the presence of bile. Appl Environ Microbiol. 2002,68(8):3855-8, doi: 10.1128/aem.68.8.3855-3858.2002.

33. Crawford RW, Gibson DL, Kay WW, Gunn JS. Identification of a bile-induced exopolysaccharide required for Salmonella biofilm formation on gallstone surfaces. Infect Immun. 2008,76(11):5341-9, doi: 10.1128/IAl.00786-08.

34. Hernández SB, Cota I, Ducret A, Aussel L, Casadesús J. Adaptation and Preadaptation of Salmonella enterica to Bile. PLOS Genetics. 2012,8(1):e1002459, doi: 10.1371/journal.pgen.1002459.

35. Urdaneta V, Casadesús J. Interactions between Bacteria and Bile Salts in the Gastrointestinal and Hepatobiliary Tracts. Frontiers in Medicine. 2017,4(163), doi: 10.3389/fmed.2017.00163.

36. Wahlström A, Sayin Sama I, Marschall H-U, Bäckhed F. Intestinal Crosstalk between Bile Acids and Microbiota and Its Impact on Host Metabolism. Cell Metabolism. 2016,24(1):41-50, doi: 10.1016/j.cmet.2016.05.005.

37. Belzer C, Kusters JG, Kuipers EJ, van Vliet AHM. Urease induced calcium precipitation by Helicobacter species may initiate gallstone formation. Gut. 2006,55(11):1678-9, doi: 10.1136/gut.2006.098319.

38. JAHANI SHERAFAT S. ET, M. REZA SEYYED MAJIDI, F. VAZIRI, M. ALEBOUYEH, A.H. MOHAMMAD ALIZADEH, E. NAZEMALHOSSEINI MOJARAD and M. REZA ZALI. Lack of Association between Helicobacter pylori Infection and Biliary Tract Diseases. 2012,61(4):319-22.

39. Monstein HJ, Jonsson Y, Zdolsek J, Svanvik J. Identification of Helicobacter pylori DNA in Human Cholesterol Gallstones. Scandinavian Journal of Gastroenterology. 2002,37(1):112-9, doi: 10.1080/003655202753387455.

40. Fatemi SM, Doosti A, Shokri D, Ghorbani-Dalini S, Molazadeh M, Tavakoli H, et al. Is There a Correlation between Helicobacter Pylori and Enterohepatic Helicobacter Species and Gallstone Cholecystitis? Middle East J Dig Dis. 2018,10(1):24-30, doi: 10.15171/mejdd.2017.86.

41. Mima K, Nakagawa S, Sawayama H, Ishimoto T, Imai K, Iwatsuki M, et al. The microbiome and hepatobiliary-pancreatic cancers. Cancer Letters. 2017,402:9-15, doi: https://doi.org/10.1016/j.canlet.2017.05.001.

42. Dar MY, Ali, S., Raina, A.H. et al. Indian J Gastroenterol (2016) 35: 343. . Association of Helicobacter pylori with hepatobiliary stone disease, a prospective case control study. Indian Journal of Gastroenterology. 2016,35(5):343-6.

43. Skar V SA, Strømme JH. Beta-glucuronidase activity related to bacterial growth in common bile duct bile in gallstone patients. Scand J Gastroenterol Jan,23(1):83-90.1988 23(1):83-90.

44. Peng Y, Yang Y, Liu Y, Nie Y, Xu P, Xia B, et al. Cholesterol gallstones and bile host diverse bacterial communities with potential to promote the formation of gallstones. Microb Pathog. 2015,83-84:57-63, doi: 10.1016/j.micpath.2015.05.002.

45. Saeb ATM. Presence of Bacterial Virulence Gene Homologues in the dibenzo-p-dioxins degrading bacterium Sphingomonas wittichii. Bioinformation. 2016,12(4):241-8, doi: 10.6026/97320630012241.

46. Liang T, Su W, Zhang Q, Li G, Gao S, Lou J, et al. Roles of Sphincter of Oddi Laxity in Bile Duct Microenvironment in Patients with Cholangiolithiasis: From the Perspective of the Microbiome and Metabolome. Journal of the American College of Surgeons. 2016,222(3):269-80.e10, doi: 10.1016/j.jamcollsurg.2015.12.009.

47. Saltykova IV, Petrov VA, Logacheva MD, Ivanova PG, Merzlikin NV, Sazonov AE, et al. Biliary Microbiota, Gallstone Disease and Infection with Opisthorchis felineus. PLOS Neglected Tropical Diseases. 2016,10(7):e0004809, doi: 10.1371/journal.pntd.0004809.

\section{Figures}




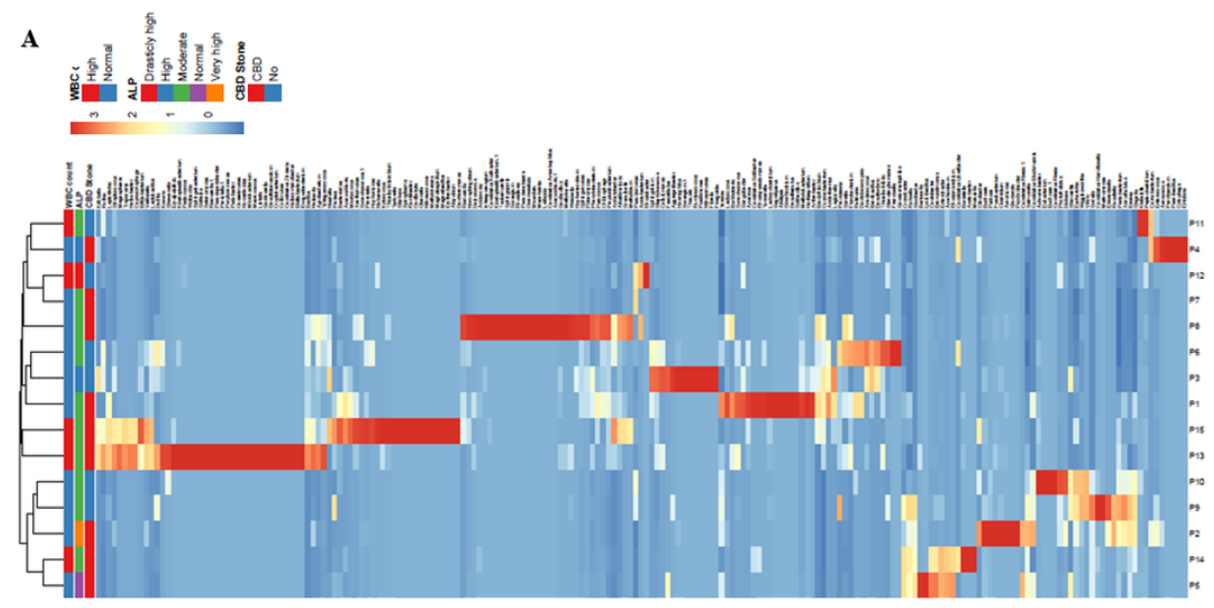

B

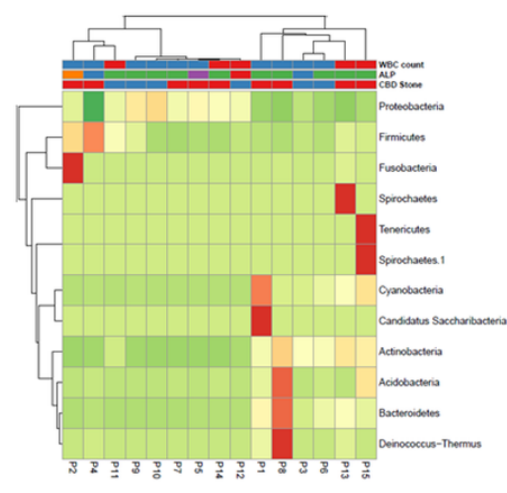

C

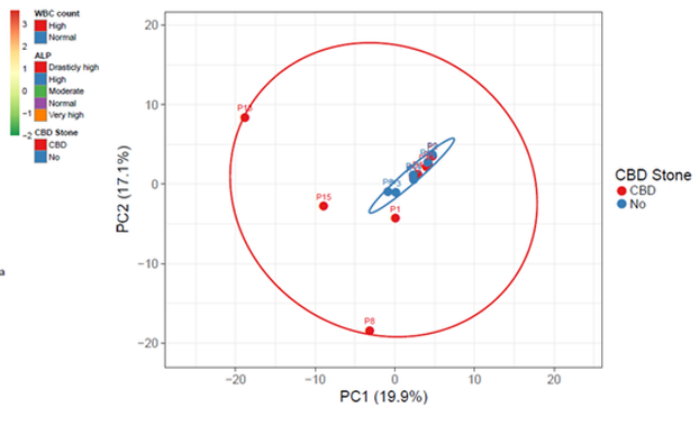

\section{Figure 1}

Heatmap and clustering for multivariable data of bacterial genera (A) and phyla (B) characterized in bile samples of patients with biliary diseases. Communities of the bacterial genera were clustered using Principal Coordinates Analysis (PCoA) of the unweighted UniFrac distance matrix (C). Unit variance scaling was applied to rows; SVD with imputation is used to calculate principal components. $\mathrm{X}$ and $\mathrm{Y}$ axis show principal component 1 (PC1) and principal component 2 (PC2) that explain $19.9 \%$ and $17.1 \%$ of the total variance, respectively. $\mathrm{N}=15$ data points. Each point corresponds to a community colored according to existence of $\mathrm{CBD}$ (red, Yes; blue No). The percentage of variation explained by the plotted principal coordinates is indicated on the axes.

\section{Supplementary Files}

This is a list of supplementary files associated with this preprint. Click to download.

- Additionalfiles.docx 